\title{
Profissionais da saúde e as condições de biossegurança no enfretamento da COVID-19
}

\author{
Health professionals and biosafety conditions in coping with COVID-19
}

Profesionales de la salud y condiciones de bioseguridad frente al COVID-19

Recebido: 11/09/2021 | Revisado: 17/09/2021 | Aceito: 21/09/2021 | Publicado: 23/09/2021

Lucas Daniel Souza de Vasconcelos

ORCID: https://orcid.org/0000-0002-9628-6431 Universidade Estadual de Ciências da Saúde de Alagoas, Brasil

E-mail: danielvasconcelosfono@ gmail.com

Anderson da Silva Moreira

ORCID: https://orcid.org/0000-0003-1961-6262 Universidade Estadual de Ciências da Saúde de Alagoas, Brasil

E-mail: anderson.moreira@academico.uncisal.edu.br

Juliana Maria Bulhões Ferreira

ORCID: https://orcid.org/0000-0002-0633-9350 Universidade Estadual de Ciências da Saúde de Alagoas, Brasil

E-mail: juliana.ferreira@academico.uncisal.edu.br

Yasmin Mainique Leite Gomes ORCID: https://orcid.org/0000-0002-6209-2137 Universidade Estadual de Ciências da Saúde de Alagoas, Brasil E-mail: yasmin.gomes@academico.uncisal.edu.br

Ranilde Cristiane Cavalcante Costa

ORCID: https://orcid.org/0000-0001-6877-3307

Universidade Estadual de Ciências da Saúde de Alagoas, Brasil

E-mail: ranilde.costa@academico.uncisal.edu.br

John Victor dos Santos Silva

ORCID: https://orcid.org/0000-0003-4671-102X

Universidade de São Paulo, Brasil

E-mail: john.setedejulho@gmail.com

Géssyca Cavalcante de Melo

ORCID: https://orcid.org/0000-0002-6774-857X

Universidade Estadual de Ciências da Saúde de Alagoas, Brasil

E-mail: gessyca.melo@ academico.uncisal.edu.br

Vanessa Fernandes de Almeida Porto

ORCID: https://orcid.org/0000-0002-0100-4869

Universidade Estadual de Ciências da Saúde de Alagoas, Brasil

E-mail: vanessa.porto@academico.uncisal.edu.br

\begin{abstract}
Resumo
Objetivo: caracterizar os profissionais de saúde e suas condições de biossegurança no enfrentamento da pandemia da COVID-19 em Maceió-AL. Metodologia: estudo descritivo, de delineamento transversal, com abordagem quantitativa e técnica de amostragem não probabilística intencional. Foi aplicado um questionário eletrônico, elaborado por meio da plataforma Google Forms, com variáveis sociodemográficas do perfil profissional e perguntas que abordavam opiniões e situações do trabalho na pandemia. O questionário foi compartilhado pelos aplicativos de mensagens, redes sociais e e-mail. Resultados: participaram da pesquisa 255 profissionais da saúde de diversas áreas de atuação. A maioria dos profissionais são mulheres jovens com formação profissional em diversas categorias, principalmente enfermagem. Os profissionais em sua maioria possuem dois empregos, com tempo de formação menor que cinco anos, carga horária semanal de 60 ou mais horas e trabalham predominantemente em instituições públicas e no serviço hospitalar. Entre os profissionais, $42 \%$ já apresentaram sintomas gripais ou confirmação de COVID-19. Acerca das condições de trabalho, a $88 \%$ receberam EPIs e orientações sobre biossegurança nos seus locais de trabalho, sendo a máscara cirúrgica usada com maior frequência. Conclusão: foram observadas boas condições para realização do trabalho em saúde, entretanto, é necessário traçar estratégias de enfrentamento que levem em conta as singularidades de cada profissão e o grau de exposição da categoria.
\end{abstract}

Palavras-chave: COVID-19; SARS-CoV-2; Pessoal de saúde; Condições de trabalho.

\section{Abstract}

Objective: to characterize health professionals and biosafety conditions in coping with COVID-19 in Maceió- Al. Methodology: descriptive, cross-sectional study, with a quantitative approach and intentional non-probabilistic sampling technique. An electronic questionnaire was applied, elaborated through the Google Forms platform, with sociodemographic variables of the professional profile and questions that addressed opinions and work situations in the 
pandemic. The questionnaire was shared by messaging, social media and email apps. Results: 255 health professionals from different areas participated in the survey. Most professionals are young women with professional training in different categories, especially nursing. Most professionals have two jobs, with less than five years of training, a weekly workload of 60 or more hours and work predominantly in public institutions and in the hospital service. Among professionals, $42 \%$ have already had flu-like symptoms or confirmation of COVID-19. Regarding working conditions, $88 \%$ received PPE and guidance on biosafety in their workplaces, with the surgical mask being used most frequently. Conclusion: good conditions were observed for carrying out health work, however, it is necessary to outline coping strategies that take into account the singularities of each profession and the degree of exposure of the category.

Keywords: COVID-19; SARS-CoV-2; Health personnel; Working conditions.

\section{Resumen}

Objetivo: caracterizar a los profesionales de la salud y las condiciones de bioseguridad en el afrontamiento del COVID19 em Maceió-al. Metodología: estudio descriptivo, transversal, con abordaje cuantitativo y técnica de muestreo intencional no probabilístico. Se aplicó un cuestionario electrónico, elaborado a través de la plataforma Google Forms, con variables sociodemográficas del perfil profesional y preguntas que abordaban opiniones y situaciones laborales en la pandemia. El cuestionario fue compartido por mensajería, redes sociales y aplicaciones de correo electrónico. Resultados: Participaron de la encuesta 255 profesionales de la salud de diferentes áreas. La mayoría de los profesionales son mujeres jóvenes con formación profesional en diferentes categorías, especialmente enfermería. La mayoría de los profesionales tienen dos puestos de trabajo, con menos de cinco años de formación, una carga de trabajo semanal de 60 o más horas y laboran predominantemente en instituciones públicas y en el servicio hospitalario. Entre los profesionales, el $42 \%$ ya ha tenido síntomas similares a los de la gripe o confirmación de COVID-19. En cuanto a las condiciones laborales, el 88\% recibió EPP y orientación sobre bioseguridad en sus lugares de trabajo, siendo la mascarilla quirúrgica la que se utiliza con mayor frecuencia. Conclusión: se observaron buenas condiciones para realizar el trabajo de salud, sin embargo, es necesario delinear estrategias de afrontamiento que tengan en cuenta las singularidades de cada profesión y el grado de exposición de la categoría.

Palabras clave: COVID-19; SARS-CoV-2; Personal sanitario; Las condiciones de trabajo.

\section{Introdução}

No ano de 2020, uma emergência global caracterizada como uma pandemia ocasionada pelo novo coronavírus SARSCoV-2 (Síndrome Respiratória Aguda Grave), denominada como Corona Virus Disease (COVID-19), provocou mudanças na rotina dos profissionais de saúde, exigindo o desenvolvimento de estratégias voltadas para a saúde e segurança destes profissionais, tendo em vista a exposição ao vírus durante as atividades laborais (Santos Filho et al., 2020).

$\mathrm{O}$ vírus SARS-CoV-2 pertence à espécie SARS-like coronavírus e apresenta disseminação maior que os outros da mesma espécie, com relatos de que cada doente pode infectar de dois a três outros indivíduos (Wang, 2020).

A transmissão ocorre pelo contato próximo e desprotegido com secreções ou excreções de um paciente infectado, a exemplo de sangue, fezes, vômitos, urina e principalmente por meio de gotículas salivares (Hossain, 2020), sendo a utilização de Equipamentos de Proteção Individual (EPIs) a medida disponível para minimizar o risco de contaminação dos profissionais da saúde. Contudo, no caso da COVID- 19, a eficácia do EPI está associada não apenas ao fornecimento de equipamentos, mas também ao treinamento adequado das equipes de trabalhadores para o uso correto e consistente (Lai, 2020).

A Agência Nacional de Vigilância Sanitária (ANVISA) do Brasil, responsável por fornecer orientações para prevenção e controle da infecção pelo novo coronavírus, em sua nota técnica n ${ }^{\circ}$ 04/2020, atualizada em 21 de março de 2020, recomenda que as políticas e práticas organizacionais minimizem a exposição dos profissionais de saúde ao SARS-CoV-2 no atendimento pré-hospitalar e dentro dos serviços de saúde. Ademais, preconiza a higienização das mãos com água e sabonete líquido ou preparação alcoólica a 70\%, assim como o uso de óculos de proteção ou protetor facial, uso de máscara cirúrgica, avental impermeável e luvas de procedimento (Brasil, 2020a).

Associado a isso, o uso de gorro e máscara N95 ou PFF2 é indicado durante a realização de procedimentos geradores de aerossóis, como intubação ou aspiração traqueal, ventilação mecânica invasiva e não invasiva, ressuscitação cardiopulmonar, ventilação manual antes da intubação e coletas de amostras oronasofaríngea para análise (ANVISA, 2020). 
Embora as recomendações sobre a utilização de EPIs tenham sido estabelecidas desde o início da pandemia, na prática, a escassez de equipamentos tem sido a realidade de diversos países, propiciando, um risco iminente à saúde do trabalhador por contaminação decorrente da exposição desprotegida (Adhikari, 2020; Newman, 2020).

À vista disso, sabe-se que a pandemia vem afetando tanto as condições de vida, quanto as de trabalho de milhões de pessoas em todo o mundo, sobretudo pelo aumento do desemprego e pela precarização das condições de trabalho. Desse modo, a pandemia contribui para explicitar as imensas fragilidades das sociedades, exibidas nas condições laborais às quais os trabalhadores são submetidos (Leite, 2020).

Mesmo diante da maior exposição dos profissionais da saúde, até o presente momento, há poucos estudos que apresentem números quanto à contaminação, curados e óbitos destes trabalhadores, se estão recebendo os EPIs, quais as condições de trabalho durante a pandemia ou se recebem, por exemplo, orientações adequadas para os momentos em que estão expostos nos serviços de atendimento (Filho et al.,2020).

Diante do exposto, este estudo tem como objetivo caracterizar os profissionais da saúde e as condições de biossegurança no enfretamento da COVID-19 em Maceió-AL.

\section{Metodologia}

Trata-se de um estudo descritivo, de delineamento transversal, com abordagem quantitativa. Os profissionais foram selecionados através da técnica de amostragem de bola de neve de Vinuto (2014), sendo os participantes que conheciam os pesquisadores nomeados como "sementes". Esses participaram do estudo divulgando a investigação com profissionais conhecidos.

Inicialmente, os profissionais receberam orientações por escrito quanto aos objetivos e relevância social da investigação e, aqueles que aceitaram participar da pesquisa de forma voluntária, assinaram o Termo de Consentimento Livre e Esclarecido (TCLE) e responderam ao questionário eletrônico.

Participaram do estudo profissionais de saúde que atuaram no enfrentamento da pandemia de COVID-19, seja no âmbito da atenção primária e/ou no âmbito hospitalar no município de Maceió/AL. Os critérios de inclusão para participar da pesquisa foram: ser profissional de saúde que estava atuando no atendimento a pessoas com suspeitas, diagnóstico e/ou realizando tratamento de COVID-19 na capital alagoana. Foram excluídos aqueles que estavam em licença médica e/ou afastados nesse período da pandemia de COVID-19 durante o período de coleta de dados.

Os dados foram coletados no período de agosto a setembro de 2020. Para a divulgação do estudo foi concedida entrevista no jornal local de uma emissora de televisão, publicação de folders nas redes sociais da universidade e dos pesquisadores.

O instrumento de coleta de dados consistiu em um questionário eletrônico, criado por meio da plataforma Google Forms, elaborado pelos pesquisadores e baseado nos estudos de Lima et al. (2017), Pereira e Borba (2016), Barbosa et al. (2017) e Pereira et al. (2017). O questionário foi composto por perguntas que abordavam opiniões e situações cotidianas do trabalho, tais como: biossegurança e uso de EPIs na rotina laboral (questões de recebimento de orientações acerca do uso de EPI e contato com superfícies próximas ao paciente e outros) e as condições de trabalho na pandemia (questões acerca da estrutura do serviço, insumos básicos, recebimento de auxílio remuneração e outras).

Além disso, para atender aos objetivos propostos foram adicionadas ao questionário as variáveis sociodemográficas: idade, sexo, profissão, tempo de formação, quantidade de empregos e carga horária semanal. As perguntas foram respondidas com "Sim", "Não", "Não desejo responder" ou com alguma alternativa específica relacionada à pergunta. Os profissionais foram contatados de maneira intencional, sendo esses da rede de contatos pessoais dos pesquisadores e em contato com preceptores em hospitais e unidades de saúde com vínculo com a universidade. O questionário eletrônico foi compartilhado pelos aplicativos de mensagens, redes sociais e e-mail. 
Os dados foram coletados em um formulário padronizado e os mesmos armazenados em uma planilha eletrônica de dados (Microsoft Excel® 2013. Redmond, WA, EUA). Em que, cada linha correspondeu a um sujeito de pesquisa/formulário e cada coluna às informações obtidas na coleta. As informações foram analisadas por meio de estatística descritiva com frequência absoluta e percentuais, enquanto que os resultados foram apresentados em quadros, gráficos e/ou tabelas.

O presente estudo foi aprovado pelo Comitê de Ética em Pesquisa da Universidade Estadual de Ciências da Saúde de Alagoas - UNCISAL sob o número de CAAE: 34565020.5.0000.5011.

\section{Resultados}

Participaram da pesquisa 255 profissionais da saúde, destes $72,5 \%$ são mulheres, com idade entre 20 e maior que 40 anos. Os profissionais possuíam formação em diversas áreas, sendo as predominantes a enfermagem (29,0\%), medicina (14,9\%), fisioterapia $(9,8 \%)$ e técnico de enfermagem (18,4\%). Em relação a carga horária semanal e a quantidade de empregos, 34,6\% dos profissionais tinham jornada de trabalho de 60 horas ou mais por semana e 46,3\% dos participantes possuíam 2 empregos.

A Tabela 1 apresenta a caracterização dos participantes quanto aos dados do perfil sociodemográficos.

Tabela 1 - Características sociodemográficas dos profissionais de saúde. Maceió, AL, 2021.

\begin{tabular}{|c|c|c|}
\hline Variáveis do estudo & $\mathbf{N}$ & $\%$ \\
\hline \multicolumn{3}{|l|}{ Sexo } \\
\hline Feminino & 185 & 72,5 \\
\hline Masculino & 69 & 27,1 \\
\hline Não desejo responder & 1 & 0,04 \\
\hline \multicolumn{3}{|l|}{ Faixa Etária } \\
\hline 20 a 30 anos & 83 & 32,5 \\
\hline 31 a 40 anos & 92 & 38,4 \\
\hline 41 a 50 anos & 53 & 20,8 \\
\hline$>50$ anos & 21 & 8,2 \\
\hline Não desejo responder & 6 & 0,1 \\
\hline \multicolumn{3}{|l|}{ Formação profissional } \\
\hline Enfermagem & 74 & 29,0 \\
\hline Fisioterapia & 25 & 9,8 \\
\hline Fonoaudiologia & 11 & 4,3 \\
\hline Medicina & 38 & 14,9 \\
\hline Terapia Ocupacional & 12 & 4,7 \\
\hline Nutrição & 4 & 1,6 \\
\hline Odontologia & 10 & 3,9 \\
\hline Psicologia & 6 & 2,4 \\
\hline Biomedicina & 7 & 2,7 \\
\hline Assistente Social & 4 & 1,6 \\
\hline Técnico de Enfermagem & 47 & 18,4 \\
\hline Outros & 8 & 3,2 \\
\hline Não desejo responder & 1 & 0,4 \\
\hline
\end{tabular}




\section{Tempo de formação}

$<5$ anos 79

31,0

Entre 5 e 10 anos

74

29,0

Entre 11 e 20 anos

67

26,3

$>20$ anos

33

12,9

Não desejo responder

2

\section{Quantidade de empregos}

1 emprego

2 empregos

3 ou mais empregos

49

19,2

Não desejo responder

5

\section{Carga horária semanal}

Até $20 \mathrm{~h}$

$$
6
$$

De 21 às $40 \mathrm{~h}$

$41 \mathrm{~h}$ às $50 \mathrm{~h}$

$60 \mathrm{~h}$ ou mais

89 34,9

Não desejo responder

\section{Tipo de Instituição}

Pública 115

Privada

Ambas

77

30,2

Não desejo responder

\section{Tipo de Serviço}

Hospitalar 168

Atenção Primária

Ambas

Não desejo responder

Fonte: Dados da pesquisa (2021).

Em relação sobre as orientações acerca da biossegurança, 50,0\% dos participantes não receberam informações de como inspecionar visualmente a máscara N95 ou equivalente, antes de cada uso, com a finalidade de verificar sua integridade. Ainda, 24,0\% não receberam orientações de como remover o EPI supracitado com segurança. A Tabela 2 apresenta os dados gerais de orientações quanto aos cuidados com biossegurança e uso de EPIs que os profissionais receberam em seus locais de trabalho. 
Tabela 2 - Orientações sobre biosegurança. Maceió, AL, 2021.

\begin{tabular}{|c|c|c|c|}
\hline Recebeu orientações sobre & SIM & NÃO & NDR \\
\hline Higienização das mãos & $91 \%$ & $9 \%$ & $0 \%$ \\
\hline Evitar tocar olhos, nariz e boca & $94 \%$ & $6 \%$ & $0 \%$ \\
\hline Evitar tocar superfícies próximas ao paciente & $89 \%$ & $11 \%$ & $0 \%$ \\
\hline Medidas de precaução a serem adotadas & $91 \%$ & $8 \%$ & $1 \%$ \\
\hline Utilizar Equipamentos de Proteção Individual (EPI) & $93 \%$ & $7 \%$ & $0 \%$ \\
\hline $\begin{array}{l}\text { Inspecionar visualmente a máscara N95 ou equivalente, antes de cada uso, para verificar sua } \\
\text { integridade. }\end{array}$ & $49 \%$ & $50 \%$ & $1 \%$ \\
\hline Como remover a máscara N95 ou equivalente & $76 \%$ & $24 \%$ & $0 \%$ \\
\hline
\end{tabular}

Fonte: Dados da pesquisa (2021).

Sobre os EPIs, $88 \%$ dos profissionais receberam nos seus locais de trabalho e $60 \%$ referem que se sentem seguros com o seu uso. A Figura 1 apresenta os EPIs que os profissionais receberam para exercer as atividades durante o enfrentamento da pandemia.

Figura 1 - Relação de EPIs que os profissionais receberam. Maceió, AL, 2021.

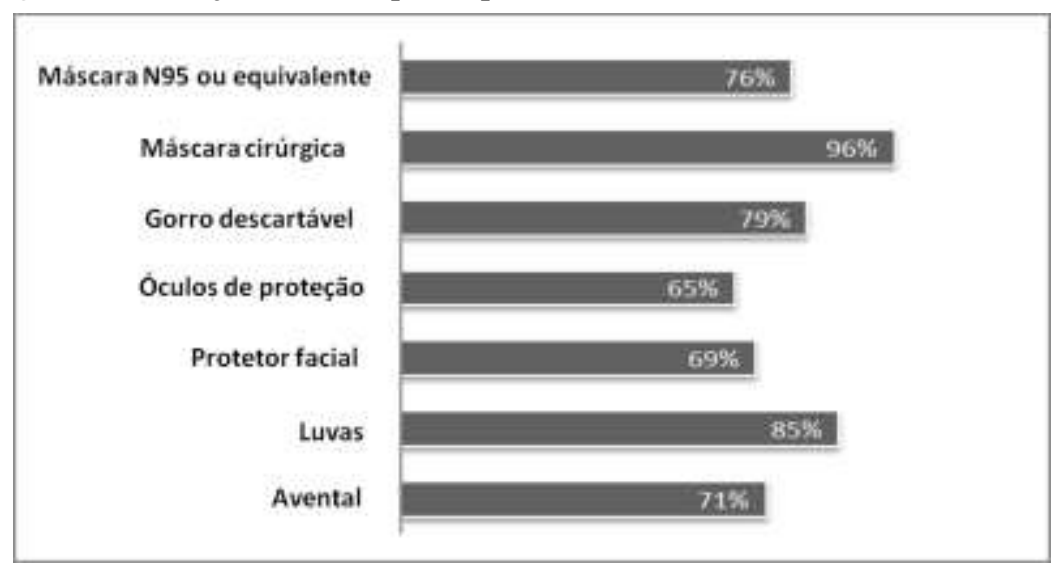

Fonte: Dados da pesquisa (2021).

Sobre o recebimento de incentivo ou auxílio em remuneração para exercer sua atividade profissional contra o COVID19, 78\% dos profissionais não receberam, enquanto 22\% afirmam ter recebido esse estímulo para o exercício de sua atividade laboral. Desses profissionais, 29 (59\%) atuam em serviços hospitalares, 14 (29\%) trabalham em serviços de atenção primária e $06(12 \%)$ atuam em ambos os serviços. 25 (51\%) profissionais atuam em instituições públicas, 07 (14\%) profissionais nas instituições privadas e 17 (35\%) profissionais em ambas as instituições. Além disso, ressalta-se que profissionais de 10 categorias diferentes afirmam terem sido contemplados com o incentivo, como técnicos de enfermagem, fonoaudiólogos, terapeutas ocupacionais, nutricionistas e outros.

No que se refere à disponibilidade de insumos básicos de trabalho, como sabão líquido, papel toalha e álcool $70 \%$ em gel/líquido, $90 \%$ dos profissionais referem que existiam todos esses insumos em sua unidade de trabalho, no entanto, 22 afirmaram a ausência desses materiais no seu local de atuação, sendo 13 profissionais atuantes em instituições públicas e 09 em ambas as instituições (públicas e privadas).

No que se refere à presença de locais adequados para descarte de lixo e materiais infectados, $88 \%$ dos profissionais referiram haver em sua unidade de trabalho espaço adequado para esse descarte, enquanto 11\% relataram que não há esse lugar apropriado no seu local de atuação. 
A Figura 2 apresenta o percentual dos profissionais de saúde que apresentaram diagnóstico confirmado ou sintomas sugestivos de COVID-19.

Figura 2 - Profissional que já apresentaram sintomas ou diagnóstico confirmado de COVID-19.

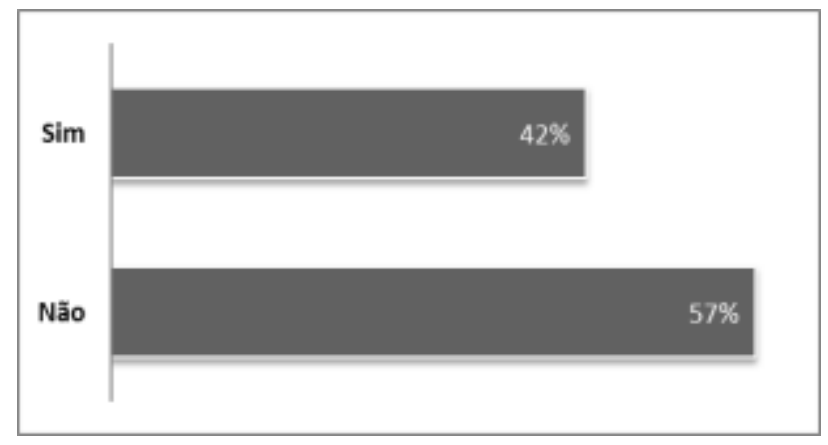

Fonte: Dados da pesquisa (2021).

\section{Discussão}

A pandemia de COVID-19 é um desafio sem precedentes para a ciência. O cenário de baixa produção/distribuição de vacinas (ressalta-se que a vacinação ainda não havia iniciado no Brasil no período da pesquisa) associado à ausência de medicamentos específicos para o tratamento da doença, evidenciou a necessidade de adotar-se medidas rápidas e eficazes que pudessem interromper a cadeia de transmissibilidade do vírus. Desse modo, o isolamento, bem como o distanciamento social mostraram-se eficientes no controle da velocidade e da expansão da pandemia (Aquino et al., 2020).

Nesse sentido, neste trabalho constatou-se a predominância de profissionais do sexo feminino, corroborando achados de outras pesquisas (Borges \& Bianchin, 2015; Paschoa, Zanei \& Whitaker, 2007; Santos \& Beresin, 2009) ao afirmar que a promoção do cuidado em saúde é protagonizada por mulheres. Segundo Wermelinger et al (2010), na área da saúde, a participação feminina chega a quase $70 \%$ do total de trabalhadores, com $62 \%$ da força de trabalho das categorias profissionais de nível superior, atingindo 74\% nos estratos profissionais de nível médio. Sendo assim, esse estudo reafirma os dados do Fundo de População das Nações Unidas - UNFPA (2020), quando apontam que em todo o mundo as mulheres representam 70\% das equipes de profissionais de serviços sociais e de saúde.

A predominância de jovens entre os profissionais que atuam no enfretamento da pandemia da COVID-19 encontrada na presente pesquisa está em consonância com outros estudos da área (Tottoli et al., 2019; Borges \& Bianchin, 2015; Ribeiro et al., 2014) que apontam um maior número de profissionais da saúde no intervalo na faixa etária entre 20 a 40 anos de idade.

Diferentes categorias da área da saúde participaram da presente pesquisa, com destaque para enfermagem, medicina e fisioterapia. Essa tendência é observada em uma pesquisa realizada pela Fundação Oswaldo Cruz (Leonel, 2021), demonstrando um reflexo nacional do perfil profissional encontrado neste estudo. Vale ressaltar que o período pandêmico trouxe a valorização dos trabalhadores da saúde, isto é, fez com que outros profissionais de saúde, além de médicos, ganhassem maior notoriedade, como é o caso das categorias acima citadas (Duarte, Silva \& Bagatini, 2021).

Dessa forma, dados deste estudo apontam que a Enfermagem é a categoria com maior número de profissionais em trabalho, tanto em serviços hospitalares quanto na atenção primária. $\mathrm{O}$ fato é que esses profissionais estão potencialmente expostos durante o enfrentamento da pandemia de COVID-19, sobretudo pelo fato de estarem presentes em diferentes ambientes de assistência à saúde e serem os profissionais que trabalham em contato direto e cotidianamente com os pacientes (Teixeira et al., 2020). 
Vale ressaltar, entretanto, que a composição profissional das equipes de saúde deve priorizar a interprofissionalidade, objetivando a implementação integral da assistência à saúde, possibilitando uma visão ampla do usuário e uma melhor resolução do caso (Brasil, 2018a). Durante a pandemia de COVID-19, Tanaka e colaboradores (2020) destacam que as ações realizadas em equipe contribuíram para melhorar a prática dos profissionais da saúde na assistência, fortalecendo o vínculo multiprofissional e um cuidado mais qualificado, seguro e humanizado.

A rede pública de saúde reúne a maior parte dos profissionais que participaram deste estudo. Esse quantitativo de profissionais deve atender aos usuários do SUS, visto que dados do Instituto Brasileiro de Geografia e Estatística (IBGE) de 2013 mostram que a maior parcela das pessoas (47,9\%) procura a Unidade Básica de Saúde como o estabelecimento de referência quando necessitavam do atendimento de saúde (Brasil, 2015). Esses dados revelam a necessidade de ampliação dos serviços de saúde, considerando que eles atendem a maior parcela da população no enfrentamento da pandemia.

A pesquisa contou com grande participação de profissionais que atuam na rede de atenção hospitalar, local esse que necessitou da maior oferta de vagas para os trabalhadores, visando suprir a demanda de atendimentos à população neste período de aumento e gravidade dos casos. Segundo Campos, Canabrava (2020) a pandemia possibilitou visualizar melhor a estrutura assistencial hospitalar brasileira que é historicamente insuficiente e severamente desgastada pelo subfinanciamento crônico. Tal fato pode justificar o impacto recebido pela grande demanda de leitos hospitalares (gerais e de Unidades de Terapia Intensiva UTI) para o cuidado às vítimas da COVID-19, sobretudo as mais graves. Nesse cenário, algumas medidas podem contribuir para ampliar a oferta dos serviços hospitalares, ocorrendo em Maceió, a construção de hospitais de campanha e de serviços específicos no diagnóstico e no tratamento da COVID-19.

A Atenção Primária à Saúde (APS) contribui para as atividades no enfrentamento ao COVID-19 nesse tipo de serviço. Segundo Giovanella et al (2020), esse é o espaço em que os profissionais conhecem seus territórios, sua comunidade, suas vulnerabilidades e, em geral, atuam na perspectiva da vigilância em saúde, principalmente no controle do contágio e na orientação comunitária quanto à necessidade da manutenção do isolamento social. Além disso, a APS representa um importante papel na continuidade do cuidado aos pacientes com COVID-19 (Medina et al., 2020).

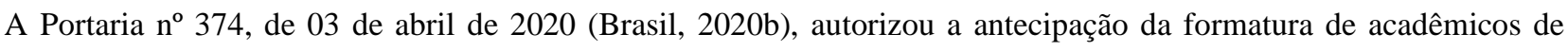
Enfermagem, Fisioterapia, Medicina e outros cursos, objetivando contribuir com o corpo de profissionais para atuar no enfrentamento da doença durante a pandemia. Sendo assim, essa decisão contribuiu de forma substancial para o aumento no número de profissionais com menos de cinco anos de formado atuando no enfrentamento ao COVID-19.

Dados referentes à carga horária e quantidade de vínculo empregatício revelam que os profissionais de saúde estão expostos a extensas jornadas semanais, associado ao tempo gasto com deslocamentos entre os locais de trabalho. Dessa maneira, o cansaço físico, o estresse psicológico, medo da contaminação, insuficiência e/ou negligência com relação às medidas de proteção e cuidados de saúde são relatados como aspectos que podem contribuir para uma maior suscetibilidade ao contágio desses profissionais. Aliado a todos esses fatores decorrentes do trabalho, destacam-se os fatores de interseccionalidade (sobreposição de papéis sociais e ocupações) que interagem com as posições ocupadas por mulheres na sociedade, em que estas desempenham múltiplos papéis diários, com consequente aumento das jornadas de trabalho. Assim, há a necessidade da realização de ações de promoção e proteção à saúde mental desses profissionais (Silva et al., 2020; Teixeira et al., 2020).

Quanto ao recebimento do incentivo financeiro para exercer sua atividade profissional durante a pandemia de COVID19, o pagamento dos profissionais que atuam no Sistema Único de Saúde (SUS) e no Sistema Único de Assistência Social (SUAS), assim como o auxílio ou incentivo financeiro (de acordo com os recursos dos Estados, Distrito Federal e Municípios) está previsto no inciso I, alínea "a", do artigo 5 da Lei Complementar No 173, de 27 de Maio de 2020 (Brasil, 2020d).

As máscaras têm sido uma das estratégias de biossegurança mais eficazes frente à pandemia da COVID-19. Entre os profissionais da saúde, o uso da máscara cirúrgica é frequente. Segundo o Ministério da Saúde, esse EPI deve apresentar alta 
resistência ao fluido e, obrigatoriamente, um elemento filtrante, além de necessariamente ser utilizado de forma a compreender a área do nariz e da boca. O Ministério refere-se à obrigatoriedade do uso de EPIs, bem como ao seu fornecimento a todos os profissionais, enfatizando a importância do treinamento e da orientação do manejo adequado desses equipamentos no cenário atual (Brasil, 2020c). O questionário da presente pesquisa investigou a disponibilidade e orientação quanto ao uso dos EPIs, não contemplando questões relacionadas ao tempo de utilização.

O uso adequado dos EPIs perpassa pela necessidade de definir o nível de complexidade e o tipo de serviço a ser executado. Assim, indica-se a adoção de medidas de precaução a fim de evitar o contato e a transmissão de gotículas nos casos suspeitos ou confirmados de COVID-19, bem como proteção em situações em que há exposição a aerossóis. Para isso, os profissionais da saúde em contato direto com os pacientes devem usar: batas cirúrgicas, luvas, máscara cirúrgica e proteção dos olhos (óculos de proteção ou máscara facial) (Brasil, 2020a).

Dados dispostos pela Secretaria Municipal de Saúde de Maceió, no Boletim Epidemiológico 192/2020 de 30 de setembro de 2020 informa um total de 25.109 casos de COVID-19, que representa 2,64\% da população. Em contrapartida, no presente estudo, o resultado mostra que o índice de contaminação entre os profissionais da saúde foi 15 vezes maior, quando comparado com a população. Sabe-se que o risco destes profissionais serem contaminados é superior a todas as populações, visto que estão em contato com pacientes sintomáticos ou diagnosticados com a infecção provocada pelo COVID-19.

Há muitas evidências que indicam o alto grau de exposição e contaminação dos profissionais de saúde pelo COVID-19. De acordo com os dados do Ministério de Saúde, até o dia 28 de junho de 2021, foram notificados 443.962 casos de síndrome gripal (SG) suspeitos de COVID-19, destes, 120.240 foram confirmados. Segundo os dados, as profissões de saúde com maiores registros e casos confirmados de SG por COVID-19 foram técnicos/auxiliares de enfermagem, seguidos de enfermeiros, médicos, agentes comunitários de saúde e farmacêuticos (Brasil, 2021c).

Além disso, segundo informações divulgadas pelo COFEN, em 2021, o Brasil representa um terço das mortes de profissionais de enfermagem em 44 países. Foram relatados 500 óbitos por causas relacionadas ao COVID-19, entre enfermeiros, técnicos, auxiliares de enfermagem e obstetrizes, no período entre janeiro de 2020 e janeiro de 2021 (Conselho Federal de Enfermagem, 2021).

Diante disso, é necessário traçar estratégias de enfrentamento que levem em conta as singularidades de cada profissão e o grau de exposição da categoria. Além disso, deve-se levar em conta também os determinantes que influenciam a percepção sobre saúde e doença, por serem condições que interferem na conduta adotada pelo profissional na prática de autocuidado, mesmo diante de orientações e ações de educação permanente sobre os riscos do trabalho e a necessidade de proteção.

\section{Conclusão}

A caracterização mostra que os profissionais que atuaram no enfrentamento da pandemia da COVID-19 são jovens, do sexo feminino, com pouco tempo de formação e predominantemente da área de enfermagem. Os profissionais trabalham por longas jornadas e em diferentes lugares, incluindo serviço público e privado. Muitos profissionais confirmam ter recebido EPIs e orientações quanto à biossegurança. Apesar disso, estes apresentaram sintomas ou diagnóstico relacionados à COVID-19.

\section{Referências}

Adhikari, S. P., Meng, S. Wu, Y., Mao Y., Ye, R., Wang Q., Sun, C. et al. (2020). Epidemiology, causes, clinical manifestation and diagnosis, prevention and control of coronavirus disease (COVID-19) during the early outbreak period: a scoping review. Infectious diseases of poverty, 9(29), 1-12. https://doi.org/10.1186/s40249-020-00646-x. 
Aquino, E. M. L., Silveira, I. H., Pescarini, J. M., Aquino, R., Souza-Filho, J. A., Rocha, A. S., Ferreira, A. et al. (2020). Medidas de distanciamento social no controle da pandemia de COVID-19: potenciais impactos e desafios no Brasil. Ciência \& Saúde Coletiva. 25(supl1), 2423-2446, https://doi.org/10.1590/141381232020256.1 .10502020 .

Barbosa, A. D. A., Ferreira, A. M., Martins, E. N. X., Bezerra, A. M. F., \& Bezerra, J. A. L. (2017). Percepção do enfermeiro acerca do uso de equipamentos de proteção individual em hospital paraibano. Revista Brasileira De Educação E Saúde, 7(1), 01 - 08. https://doi.org/10.18378/rebes.v7i1.4858.

Borges, T., \& Bianchini, M. (2015). Qualidade de vida dos profissionais de enfermagem de um hospital universitário do interior de São Paulo. Arquivos De Ciências Da Saúde, 22(1), 53-58. https://doi.org/10.17696/2318-3691.22.1.2015.29.

Brasil. (2015). Ministério da Saúde. Pesquisa Nacional de Saúde (PNS) 2013: acesso e utilização dos serviços de saúde, acidentes e violências: Brasil, grandes regiões e unidades da federação.

Brasil. (2018a). Ministério da Saúde. Secretaria de Atenção à Saúde Departamento de Ações Programáticas e Estratégicas. Orientações técnicas para a implementação de Linha de Cuidado para Atenção Integral à Saúde da Pessoa Idosa no Sistema Único de Saúde - SUS [recurso eletrônico].

Brasil. (2018b). Ministério da Educação. Censo da educação superior 2017.

Brasil. (2020a). Agência Nacional de Vigilância Sanitária. Orientações para serviços de saúde: medidas de prevenção e controle que devem ser adotadas durante a assistência aos casos suspeitos ou confirmados de infecção pelo novo coronavírus (SARS-COV-2).

Brasil. (2020b). Ministério da Educação. Portaria n n $^{374}$, de 3 de abril de 2020. Dispõe sobre a antecipação da colação de grau para os alunos dos cursos de Medicina, Enfermagem, Farmácia e Fisioterapia, exclusivamente para atuação nas ações de combate à pandemia do novo coronavírus - COVID-19.

Brasil. (2020c). Ministério da Saúde. Recomendações de proteção aos trabalhadores dos serviços de saúde no atendimento de COVID-19 e outras síndromes gripais.

Brasil. (2020d). Lei complementar n ${ }^{\circ}$ 173, de 27 de maio de 2020. Estabelece o Programa Federativo de Enfrentamento ao Coronavírus SARS-CoV-2 (COVID19), altera a Lei Complementar $n^{\circ} 101$, de 4 de maio de 2000, e dá outras providências.

Brasil. (2021). Ministério da Saúde. Secretaria de Vigilância em Saúde. Boletim epidemiológico especial. Doenças pelo Coronavírus COVID-19.

Campos, F. C. C. \& Canabrava, C. M. (2020). O Brasil na UTI: atenção hospitalar em tempos de pandemia. Revista Saúde em Debate, 44(Spe4), 146-160. http://dx.doi.org/10.1590/SciELOPreprints.1368.

Conselho federal de Enfermagem (Cofen). (2021). Brasil representa um terço das mortes de profissionais de Enfermagem por COVID-19. 2021. http://www.cofen.gov.br/brasil-responde-por-um-terco-das-mortes-de-profissionais- de-enfermagem-por-COVID-19_84357.html.

Duarte, M. L. C., Silva, D. G. \& Bagatini, M. M. C. (2021). Enfermagem e saúde mental: uma reflexão em meio à pandemia de coronavírus. Revista Gaúcha de Enfermagem,42(Spe.). 1-6. https://doi.org/10.1590/1983-1447.2021.20200140.

Giovanella, L. Martufi, V., Mendonza, D. C. R., Mendonça, M. H. M., Bousquat, A. E. M., Pereira, R. A. G. \& Medina, M. G. (2020). A contribuição da atenção primária à saúde na rede SUS de enfrentamento à COVID-19. Saúde em Debate, 1-21. http://dx.doi.org/10.1590/SciELOPreprints.1286.

Giovanella, L.; Franco, C. M. \& Almeida, P. F. (2020) Política Nacional de Atenção Básica: para onde vamos? Ciência \& Saúde Coletiva, 25(4), 1475-1481. https://doi.org/10.1590/1413-81232020254.01842020.

Hossain, M. M. (2020). Current status of global research on novel coronavirus disease (COVID-19): A bibliometric analysis and knowledge mapping. F1000Research. 9(374). https://papers.ssrn.com/sol3/papers.cfm?abstract_id=3547824.

Jackson Filho, J. M., Assunção, A. A., Algranti, E., Garcia, E. G., Saito, C. A. \& Maeno, M. (2020). A saúde do trabalhador e o enfrentamento da COVID-19. Revista Brasileira de Saúde Ocupacional, 45, 1-3. http://dx.doi.org/10.1590/2317-6369ed0000120.

Lai, C., Shih, T., Ko, W., Tang, H. \& Hsueh, P. (2020). Severe acute respiratory syndrome coronavirus 2 (SARS-CoV-2) and corona virus disease-2019 (COVID19): the epidemic and the challenges. International journal of antimicrobial agents, 55(3). https://doi.org/10.1016/j.ijantimicag.2020.105924.

Leonel, F. (2021). Pesquisa analisa o impacto da pandemia entre profissionais de saúde. https://portal.fiocruz.br/noticia/pesquisa-analisa-o-impacto-dapandemia-entre-profissionais-de-saude.

Lima, R. J. V., Tourinho, B. C. M. S., Costa D. S., Almeida D. M. P. F., Tapety, F. I., Landim-Almeida, C. A. P. \& Rodrigues, T. S. (2017). Agentes biológicos e equipamentos de proteção individual e coletiva: conhecimento e utilização entre profissionais. Revista prevenção de Infecção e Saúde, 3(1), 23-28. https://revistas.ufpi.br/index.php/nupcis/article/view/6684.

Medina, M. G., Giovanella, L., Bousquat, A., mendonça, M. H. M \& Aquino, R. (2020). Atenção primária à saúde em tempos de COVID-19: o que fazer? Cadernos de Saúde Pública, 36(8). http://dx.doi.org/10.1590/0102-311X00149720.

Newman, M. (2020). COVID-19: doctors' leaders warn that staff could quit and may die over lack of protective equipment. BMJ. 368, 1-2. https://doi.org/10.1136/bmj.m1257.

Paschoa, S.; Zanei, S. S. V. \& Whitaker, I. Y. (2007). Qualidade de vida dos trabalhadores de enfermagem de unidades de terapia intensiva. Acta Paulista de Enfermagem, 20(3), 305-310. https://www.scielo.br/pdf/ape/v20n3/a10v20n3.pdf.

Pereira, D. M. M., Costa, N. R., Andrade, M. N., Torres, D. C., Rocha, R. S. B. \& Avila, P. E. S. (2017). Conhecimento e adesão às práticas de biossegurança em um hospital de referência materno infantil. Reserach Medical Journal, 1(3). https://prmjournal.org/article/10.4322/prmj.2017.023/pdf/prmjournal-1-3e23.pdf. 
Pereira, M. E. C. \& Borba, C. M. (2016). Condições de trabalho e biossegurança dos profissionais que atuam na área de malacologia no Brasil. Revista Biociências, 22(2), 61-70, http://periodicos.unitau.br/ojs/index.php/biociencias/article/view/2350.

Santos Júnior, C. J., Costa, P. J. M. S., Silva, J. V. S., Souza, A. K. P., Silva, J. P., \& Rocha, T. J. M. (2020). Tecnologias digitais e de geoprocessamento aplicadas ao monitoramento da doença de coronovírus 2019 (COVID-19). Hygeia-Revista Brasileira de Geografia Médica e da Saúde, 1-10. https://doi.org/10.14393/Hygeia0053912.

Santos, R. M. A. \& Beresin, R. (2009). A qualidade de vida dos enfermeiros do centro cirúrgico. Einstein, 7(2), 152-158. http://apps.einstein.br/revista/arquivos/PDF/1214-Einsteinv7n2p152-8.pdf

Silva, D. P., Santos, I. M. R., Silva, J. V. S., Santos, M. A., \& Nascimento, Y. C. M. L. (2020). Sentimentos dos profissionais de enfermagem na saúde mental: revisão para auxiliar assistência pós novo coronavírus. Revista Científica de Enfermagem-RECIEN, 10(31), 142-154. https://doi.org/10.24276/rrecien2020.10.31.142-154.

Tanaka, A. K. S. R., Lunardi, L. S., Silva, F. G. \& Gil, L. M. C. R. (2020). O enfrentamento da equipe multidisciplinar do centro cirúrgico diante da pandemia da COVID-19. Revista Brasileira de Enfermagem, 73(sup12), 1-5. https://doi.org/10.1590/0034-7167-2020-0333.

Teixeira, C. F. S., Soares, C. M., Souza, E. A., Lisboa, E. S., Pinto, I. C. M., Andrade, L. R. \& Espiridião, M. A. (2020). A saúde dos profissionais de saúde no enfrentamento da pandemia de COVID-19. Ciência \& Saúde Coletiva, 25(9). https://doi.org/10.1590/1413-81232020259.19562020.

Tottoli, C. R., Toledo, A. M., Silva, N. C., Araújo, W. N., Souza, R. N. \& Carrego, R. L. (2019). Profissionais da saúde que atuam em ambiente hospitalar têm alta prevalência de fadiga e dorsalgia: estudo transversal. Fisioterapia \& Pesquisa, 26(1), 91-100. https://doi.org/10.1590/1809-2950/18032926012019.

United Nations Population Fund (Unfpa). (2020). COVID 19: Um olhar para gênero. http://brazil.unfpa.org/sites/default/files/pubpdf/COVID19_olhar_genero.pdf.

Wang, L., Wang, Y., Ye, D. \& Liu, Q. (2020). A review of the 2019 Novel Coronavirus (COVID-19) based on current evidence. International journal of antimicrobial agents, 55(6), 105-108. https://doi.org/10.1016/j.ijantimicag.2020.105948.

Wermelinger, M., Machado, M. H., Tavares, M. F. L., Oliveira, E. S. \& Moysés, N. M. N. (2010). A Força de Trabalho do Setor de Saúde no Brasil: Focalizando a Feminização. Revista Divulgação em Saúde para Debate, 45, 54-70. http://www.ensp.fiocruz.br/observarh/arquivos/A\%20Forca\%20de\%20Trabalho\%20d o\%20Setor\%20de\%20Saude\%20no\%20Brasil\%20.pdf.

Vinuto, J. A amostragem em bola de neve na pesquisa qualitativa: um debate em aberto. Tematicas [internet]. (2014). 22(44), 1-8. https://doi.org/10.20396/tematicas.v22i44.10977. 